\title{
Neurofibromatose de type 1: des modèles murins pour comprendre les mécanismes oncogéniques
}

L'identification de gènes impliqués dans des types spécifiques de tumeurs humaines, ainsi que le développement de nouvelles techniques de transgénèse et de recombinaison homologue permettent désormais d'obtenir des modèles animaux susceptibles de reproduire la maladie humaine. En particulier, les gènes suppresseurs de tumeurs représentent des candidats idéaux pour l'invalidation ciblée, une stratégie qui a été largement employée dans l'espoir d'obtenir un modèle murin d'un type spécifique de cancer. Les avantages de tels modèles sont multiples, car, outre le fait de favoriser la recherche et la validation de nouvelles thérapies, ils permettent des études sur les mécanismes pathogéniques qui ne peuvent pas être réalisées chez les patients. Comme la coopération oncogénique dans les cancers humains est un phénomène de plus en plus apparent, l'obtention d'animaux portant des altérations génétiques multiples s'est aussi révélée indispensable à l'analyse des interactions de gènes dans la progression tumorale. Cependant, dans la recherche contre le cancer, l'utilité des souris génétiquement modifiées dépasse le simple établissement d'un modèle de maladie humaine. L'analyse des phénotypes au cours du développement des animaux mutants homozygotes, et l'utilisation, pour des études in vitro, de lignées cellulaires dérivées de ces animaux permettent en effet d'améliorer la connaissance de la fonction normale d'un gène.

La neurofibromatose de type 1 (NF1) est une maladie génétique à transmission autosomique dominante qui

1/3500 [1]. Elle est caractérisée par le développement de neurofibromes multiples très invalidants pour le patient en raison des déformations souvent inesthétiques et des douleurs neurogènes qu'elles entraînent. I.es neurofibromes sont des tumeurs bénignes de la gaine de myéline des nerfs. Ils peuvent être cutanées, ou se développer dans les plexus des nerfs périphériques majeurs (neurofibrome plexiforme). Chez environ 5\% des patients NFl, les neurofibromes peuvent se transformer en neurofibrosarcomes, tumeurs malignes agressives fréquemment métastatiques. Des études moléculaires suggèrent que l'inactivation du gène $p 53$ joue un rôle dans cette progression sarcomateuse des neurofibromes [2]. La NF1 prédispose aussi au développement de gliomes optiques, de phéochromocytomes, et de leucémies myéloïdes juvéniles. Des manifestations non tumorales comme les taches café au lait, les lentigines dans les grands plis et les hamartomes iriens de Lisch sont aussi présentes chez les patients NF1 et contribuent au diagnostic clinique. La neurofibromine, le produit du gène $N F 1$, est un membre de la famille des protéines GAP (GTPaseactivating protein) et exerce une régulation négative sur p21 ${ }^{\text {ras }}$. La plupart des mutations du gène $N F 1$ induisent une perte de fonction de la neurofibromine, ce qui suggère la responsabilité d'une dérégulation de la voie de signalisation de $\mathrm{p} 21^{\text {ras }}$ dans la pathogénie de la NF1: on observe en effet des niveaux élevés de Ras-GTP dans les neurofibromes, les neurofibrosarcomes et les cellules leucémiques des patients NF1 $([3,4]$ et $\mathrm{m} / \mathrm{s}$ 1996, $\left.n^{\circ} 5, p .668\right)$. Dès 1971, Knudson émit l'hypothèse selon laquelle certaines tumeurs seraient dues à l'inactivation de gènes "suppresseurs de tumeurs" [5]. Cette inactivation, bi-allélique, est la conséquence de deux événements mutationnels, le premier germinal ou somatique, le second toujours somatique. En accord avec ce modèle, l'inactivation de l'allèle $N F 1$ normal a été mise en évidence dans les phéochromocytomes et dans les cellules leucémiques des patients NF1 [6, 7]. Cependant, l'inactivation bi-allélique du gène $N F 1$ n'est observée que dans quelques neurofibromes ([8] et $\mathrm{m} / \mathrm{s}$ 1995, $\left.n^{\circ} 11, p .1620\right)$.

Des études précédentes ont montré que les souris $N f 1$ hémizygotes $\left(\mathrm{Nfl}^{+/-}\right)$sont prédisposées au développement de différents types de tumeurs, principalement de fibrosarcomes, de phéochromocytomes et d'un syndrome myéloprolif eratif qui ressemble à une leucémie myélomonocytaire juvénile ([8] et $\mathrm{m} / \mathrm{s} 1995$, $n^{\circ} 1, p$. 93). Comme chez l'homme, l'analyse du génotype de ces tumeurs a souvent montré la perte de l'allèle Nf1 sauvage. Cependant, les souris $\mathrm{Nfl}^{+/-}$ne développent pas de neurofibromes, de gliomes optiques, d'hamartomes iriens de Lisch ni aucun autre signe typique de la maladie humaine. La discordance des spectres tumoraux entre homme et souris est une des limitations des modèles murins actuels d'inactivation d'un gène suppresseur de tumeurs. Plusieurs éléments peuvent contribuer à cette discordance (revue dans [10]) mais l'hypothèse la plus probable est celle d'un spectre tumoral déterminé par le taux de perte de l'allèle sauvage dans les différents compartiments cellulaires à l'origine des tumeurs. Un moyen de 
surmonter cette limitation est de créer un modèle de souris dans lequel les deux allèles du gène suppresseur de tumeurs pertinent sont inactivés. Si un tel modèle a été obtenu dans le cas particulier de la p53, la présence d'une mutation d'un gène suppresseur de tumeur à l'état homorygote s'est généralement révélée létale. C'est le cas du gène Nf1. Les souris $N / 1^{-1-}$ meurent in utero environ au jour 14 du développement et les embryons ont une hypoplasie et des malformations cardiaques [11]. La création de souris chimères comportant un contingent de cellules doublement inactivées pour le gène étudié est une voie alternative possible.

Deux équipes américaines, l'une associant des chercheurs du Massachussetts, du New Jersey et du Texas et l'autre de Dallas (TX, USA), décrivent dans le même numéro de la revue Science la mise au point de modèles de souris qui ont certaines caractéristiques de la NFl humaine et pourraient être utilisés pour tester de nouvelles stratégies thérapeutiques [12, 13]. Les deux équipes ont obtenu des souris mutantes avec différentes combinaisons alléliques d'invalidation des gènes $N f 1$ et $p 53$ et ont confirmé la coopération oncogénique des deux mutations. Ils montrent que le phénotype de ces souris, c'est-à-dire le type de tumeur développé, diffère selon la combinaison allélique et que l'on observe la perte d'hétérozygotie d'un des deux allèles voire des deux. En particulier, la mutation concomitante de $p 53$ est nécessaire au développement de tumeurs malignes de la gaine de myéline des nerfs lorsque, dans des cellules tumorales provenant de souris qui portent l'allèle muté des deux gènes sur le même chromosome, il y a perte d'hétérozygotie pour les deux locus (phénomène du co-LOH, figure 1). Grâce à l'utilisation de souris chimères comportant un contingent de cellules $\mathrm{Nfl}^{-/-}$, l'équipe dirigée par Tyler Jacks au Massachusetts Institute of Technology, MIT montre de plus que la perte de l'allèle $N f 1$ sauvage est l'élément qui limite la survenue de neurofibromes et de gliomes optiques che\% les souris $\mathrm{N} / \mathrm{I}^{+/-}$. Chez chaque souris chimère, l'analyse his-

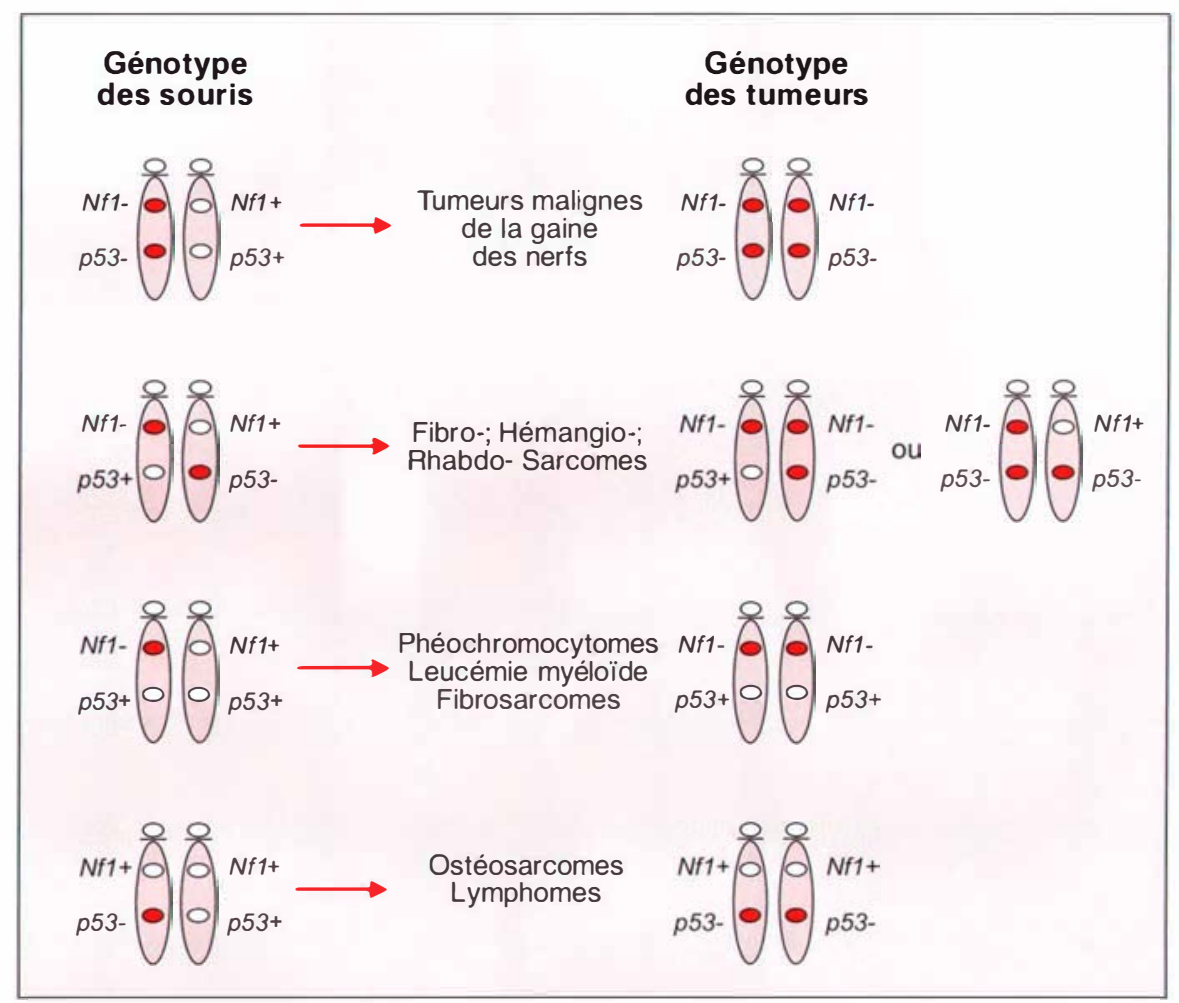

Figure 1. Différentes combinaisons alléliques des gènes Nf1 et p53 obtenues chez la souris. (en rouge) Gène invalidé, (O) gène normal. Chez la souris, les gènes Nf1 et p53 sont liés sur le chromosome 11. Les différentes combinaisons alléliques déterminent le type histologique des tumeurs dont le génotype révèle une perte d'hétérozygotie qui varie aussi selon le type de tumeur.

tologique révèle la présence d'une multitude de neurofibromes (entre 10 et 100 par souris) localisés au départ des nerfs périphériques et des ganglions des racines spinales. Ces tumeurs sont développées à partir des cellules $\mathrm{N}^{1 /-}$, et leur étude histologique et ultrastructurale révèle qu'elles sont très semblables aux neurofibromes plexiformes des patients NFl.

Il n'est cependant pas certain que ces souris chimères constituent un bon modèle pour tester ultérieurement les nouvelles thérapies géniques: elle sont difficiles à obtenir et la reproductibilité des expériences semble aléatoire étant donné que, d'une souris à l'autre et d'un tissu à l'autre, les proportions relatives de cellules normales et de cellules doublement inactivées sont très variables et difficilement contrôlables.

Une alternative serait de construire un modèle de souris dont le gène suppresseur de tumeur $N f 1$ soit inactivé sur les deux allèles, dans un lignage cellulaire déterminé, et à une période précise du développement (mutation conditionnelle ciblée sur un tissu).

1. Riccardi VM. Von Recklinghausen neurofibromatosis. NEngl J Med 1981; 305 : 1617-27.

2. Menon AG, Anderson Kill, Riccardi VM, et al. Chromosome $17 p$ deletions and p53 gene mutations associated with the formation of malignant neurofibrosarcomas in von Recklinghausen neurofibromatosis. Proc Natl Acad Sci L'SA 1990;87: 54.35-9.

3. Bollag G, Clapp IDW, Shih S, et al. Loss of NFl results in activation of the Ras signaling pathway and leads to aberrant growth in haematopoietic cells. Nat Genet 1996; 12: 1.44-8.

4. Guha A, Lau N, Huvar I, et al. Ris-GTP levels are elevated in human NFl peripheral nerve tumors. Oncogene 1996; 12: 507-13.

5. Thomas G. Dix ans de recherche sur les prédispositions génétiques au développement des tumeurs. Med Sci 1996; 11 : 336-48.

6. Xu W, Mulligan LM, Ponder MA, et al. Loss of NFl alleles in phaeochromocytomas from 
patients with type I neurofibromatosis. Genes Chrom Cancer 1992; 4 : 337-42.

7. Shannon KM, O'Connell P, Martin GA, et al. Loss of the normal NFl allele from the bone marrow of children with type 1 neurofibromatosis and malignant myeloid disorders. N Engl / Med 1994; 330: $597-601$.

8. Colman SD, W'illiams CA, W'allace MR. Benign neurofibromas in type 1 neurofibromatosis (NF1) show somatic deletions of the NF1 gene. Nal Genet $1995 ; 11: 90-2$.

9. Jacks T, Shih TS, Schmitt EM, Bronson RT, Bernards A, W'einberg RA. Tumour predisposition in mice heterozygous for a targeted mutation in Nf1. Nat (renet 1994; 7: 353-61.
10. Jacks T. Tumor suppressor gene mutations in mice. Annu Rev Genet 1996; 30: 603-36.

11. Brannan CI, Perkins AS, Vogel KS, et al. Targeted disruption of the neurofibromatosis type-1 gene leads to devclopmental abnormalities in heart and various neural crest-derived tissues [published erratum appears in Genes Der 1994; 15: 2792]. Genes Dev 1994; 8: 1019-29.

12. Cichowski K, Shih TS, Schmitt E, et al. Mouse models of tumor development in neurofibromatosis type 1 . Science $1999 ; 286$ : $2172-6$.

13. Vogel KS, Klesse LJ, Velasco-Miguel S, Meyers K, Rushing EJ, Parada L.F. Mouse tumor model for neurofibromatosis type 1. Science 1999; 286 $2176-9$

\section{Remerciements}

Je remercie R. Berger et M. Kalamarides pour a relecture de ce manuscrit.

\section{Marco Giovannini}

Inserm U.434, Fondation Jean-Dausset, CEPH, 27, rue Juliette-Dodu, 75010 Paris, France.

\section{BRÈVES}

HMGIC: une nouvelle cible anti-obésité ? Dès 1995, deux articles $\left([1,2]\right.$ et $m / s ~ 1996, n^{\circ} 8-9$, $p$. 885) ont révélé le rôle inattendu de l'un des membres de la famille des protéines HMG (High Mobility Group) connues pour leur capacité à modifier l'architecture de la chromatine. En effet, c'est l'inactivation de HMGIC par la mutation "pygmy " qui est responsable d'un phénotype de maigreur chez la souris, alors qu'à l'inverse, des réarrangements chromosomiques entraînent fréquemment un gain de fonction de cette même protéine dans les lipomes. Il s'avère maintenant que l'expression du gène Hmgci est induite spécifiquement dans le tissu adipeux, d'une part par un régime enrichi en graisse, et d'autre part chez la souris génétiquement obèse $\left(L e p^{a b} / L e p^{o b}\right)$ [3]. De plus, l'inactivation du gène Hmgic protège de l'obésité induite par le régime gras et réduit très significativement l'obésité massive des souris $L e p^{o b} / L e p^{a b}$ [3]. Cette diminution du poids de tissu adipeux est due à une réduction du nombre des cellules adipeuses. Pour expliquer ce phénomène, les auteurs suggèrent que HMGIC est nécessaire à la prolifération des pré-adipocytes, en accord avec le rôle connu de cette protéine dans les fibroblastes. En l'absence d'HMGIC, les adipocytes matures seraient moins nombreux du fait de la réduction du nombre de pré-adi- pocytes, bien que restant capables d'hypertrophie dans un contexte obésitogène. HMGIC serait-elle la cible à atteindre pour tenter de réduire l'obésité, en diminuant localement le développement du tissu adipeux ? Si tel était le cas, d'éventuels inhibiteurs d'HMGIC devraient être administrés précocement chez les sujets à risque (qui restent à déterminer !) et en complément à d'autres traitements ou régimes. En effet, bien qu'ayant une action spectaculaire sur le tissu adipeux, l'inactivation de Hmgic n'améliore pas les anomalies métaboliques associées à l'obésité !

[1. Zhou X, et al. Nature 1995; 376: 771-4.]

[2. Ashar HR, et al. Cell 1995; 82: 57-65.]

[3. Anand A, Chada K. Nat Genet $2000 ; 24: 377-80$.]

Comment modifier la sélectivité des canaux ioniques ? Une des caractéristiques des canaux ioniques est leur perméabilité plus ou moins importante aux différents ions. L'étude des mécanismes de cette sélectivité ionique, qui reposait jusqu'à récemment sur les techniques de mutagenèse dirigée, bénéficie à présent des premières données structurales $\left(m / s 2000, n^{\circ} 6 / 7, p\right.$. 823). Le groupe de $\mathrm{H}$. Bayley vient de décrire une nouvelle approche: l'utilisation d'adaptateurs [1]. On savait déjà que des oligosaccharides cycliques comme les cyclodextrines (CD) jouaient un rôle d'adaptateur pour le pore formé par une cytotoxine du staphylocoque doré, l'hémolysine $\alpha$ $(\alpha \mathrm{HL})$. Ce pore est constitué de sept sous-unités identiques assemblées dans la membrane cellulaire pour former un véritable canal faiblement sélectif pour les anions. Les CD diminuent la conductance du pore en se logeant dans le canal formé par celuici, à l'endroit même où son diamètre est le plus étroit [2]. D'où l'idée que le flux ionique passe au centre de la molécule CD nichée dans le pore, et que celle-ci puisse modifier la sélectivité ionique du pore $\alpha \mathrm{HL}$. La $\beta C D$ augmente en effet la sélectivité anionique du pore $\alpha \mathrm{HL}$ tandis que l'utilisation d'un adaptateur chargé négativement, le $\mathrm{S} 7 \beta \mathrm{CD}$, induit une sélectivité pour les cations. Si ces molécules sont placées dans un pore $\alpha H L$ mutant perméable non plus aux anions mais aux cations, elles vont aussi imposer leur propre sélectivité, anionique pour $\beta C D$ et cationique pour S7BCD [1]. On peut donc considérer ces adaptateurs comme des filtres de sélectivité qui réduisent le diamètre du canal et gouvernent sa sélectivité ionique. Cette approche pourrait s'avérer utile pour l'analyse des mécanismes de la perméabilité ionique ou pour modifier la sélectivité de certains ionophores.

[1. Gu LQ, et al. Proc Natl Acad Sci USA 2000; 97 : 3959-64.]

[2. Gu LQ, et al. Nature 1999; 398: 686-90.] 